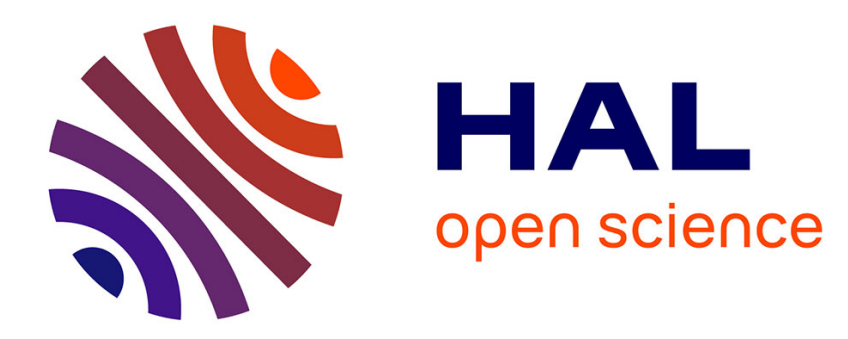

\title{
Complete quadratic Lyapunov functionals for distributed delay systems
}

\author{
Alexandre Seuret, Frédéric Gouaisbaut, Yassine Ariba
}

\section{To cite this version:}

Alexandre Seuret, Frédéric Gouaisbaut, Yassine Ariba. Complete quadratic Lyapunov functionals for distributed delay systems. Automatica, 2015, 62, pp.168-176. 10.1016/j.automatica.2015.09.030 . hal-01257679

\section{HAL Id: hal-01257679 https://hal.science/hal-01257679}

Submitted on 18 Jan 2016

HAL is a multi-disciplinary open access archive for the deposit and dissemination of scientific research documents, whether they are published or not. The documents may come from teaching and research institutions in France or abroad, or from public or private research centers.
L'archive ouverte pluridisciplinaire HAL, est destinée au dépôt et à la diffusion de documents scientifiques de niveau recherche, publiés ou non, émanant des établissements d'enseignement et de recherche français ou étrangers, des laboratoires publics ou privés. 


\title{
Complete Quadratic Lyapunov functionals for distributed delay systems
}

\author{
Alexandre Seuret ${ }^{1,2}$, Frédéric Gouaisbaut ${ }^{1,3}$, Yassine Ariba ${ }^{4,1}$
}

\begin{abstract}
This paper is concerned with the stability analysis of distributed delay systems using complete-Lyapunov functionals. Numerous articles aim at approximating their parameters thanks to a discretization method or polynomial modeling. The interest of such approximations is the design of tractable sufficient stability conditions. In the present article, we provide an alternative method based on polynomial approximation which takes advantages of the Legendre polynomials and their properties. The resulting stability conditions are scalable with respect to the maximum degree of the polynomials and are expressed in terms of tractable linear matrix inequalities. Several examples of delayed systems are tested to show the effectiveness of the method.
\end{abstract}

\section{Introduction}

The stability analysis of time-delay systems has attracted many researchers during the last decades (see for instance the survey article [23]). In particular, some of them focused on the class of distributed time delay systems, for which the delay operator, via an integral action, has a cumulative effect over the past values of the dynamics. For specific integral kernels, including constant, rationals or $\gamma$ distribution function, stability of such systems can be tackled by a direct method based on pole location $[2,13,16,22]$. For kernels which can be interpreted as impulse responses of a linear system, the system can be modeled as a linear pointwise delay system and hence classical techniques on pole location can be used (see [16]). Nevertheless, these techniques are often restricted to the case of nominal systems without uncertainties [23] and for a constant delay.

Another widely used technique for stability analysis relies on Lyapunov Theory. The construction of Lyapunov Krasovskii functionals is modified in order to take into account the distributed nature of the delay. It results some Lyapunov structure which depends on double and triple integral terms [3,5,10-12,25]. Different bounding techniques are then employed to derive numerically efficient stability tests. In general, the kernel is then restricted to be a constant function over the delay interval.

1 CNRS, LAAS, 7 avenue du Colonel Roche, 31077

Toulouse, France. \{aseuret,fgouaisb,yariba\}@laas.fr

2 Univ de Toulouse, LAAS, F-31400 Toulouse, France.

3 Univ de Toulouse, UPS, LAAS, F-31400, Toulouse, France.

4 Icam, Toulouse, France.

5 This work is supported in part by the ANR project LimICoS contract number 12 BS03 00501 and the ANR Project SCIDIS.
In order to avoid this strong constraint, another possibility, described by $\mathrm{Gu}$ et al [8], is to use a very general class of Lyapunov Krasovskii functional (see for instance [18]) and a discretization scheme to obtain numerically tractable stability conditions. This technique designed for piecewise constant delay kernel leads to a reduction of conservatism at the expense of the numerical burden. The case of general kernels has been studied successfully by [24] where the use of new integral inequalities is proposed. These new bounding techniques which were especially designed for distributed delay systems led to new Lyapunov-Krasovskii functional structures and efficient results on simulations. Another widely used approach in the literature stems from the robust analysis using the same driving forces namely the comparison systems and integral inequalities [15]. In [14], a combined full block S-Procedure and Lyapunov analysis is performed to prove the stability of a distributed delay system with a rational kernel. More recently, using quadratic separation approach, [7] has provided some Linear Matrix inequality (LMI) for polynomial kernels. In this paper, we aim at presenting a new method for the stability analysis of distributed delay systems using a Lyapunov-Krasovskii approach. The problem consists in selecting a particular structure for the LyapunovKrasovskii functional, which is composed of the sum of several classical terms [9], including a quadratic function of the instantaneous state and an integral of quadratic functional of the entire delay state. In general, all these functionals are particular cases of the well-known complete Lyapunov-Krasovskii functionals. Indeed Theorem 5.9 from [9] ensures that, if the solutions of a time delay system is asymptotically stable, this functional is a Lyapunov-Krasovskii functional, provided that its parameters satisfy some partial differential equations, which is not easy to verify, especially 
for high dimensional delay systems. In practice, numerically checking the existence of such functionals often requires an approximation of these matrix functions in an appropriate manner using the discretization method from [9] or polynomial approximation [17].

In the present article, we propose an alternative method also based on a polynomial approximation but using the particular setup of the Legendre polynomials. Thanks to these polynomials, we are able to provide a new integral inequality whose conservatism can be made arbitrarily small. This is the core tool for developing a set of new sufficient conditions indexed by $N$, equal to the degree of the polynomials modeling the parameters of the complete Lyapunov-Krasovskii functional. It is also proven that this set forms a hierarchy with respect to the pair $(h, N)$ in the sense that increasing $N$ improves the result. Finally, several examples will show the effectiveness of the method.

Notations: Throughout the paper $\mathbb{N}$ and $\mathbb{R}^{n}$ denote, the set of positive integer and the $n$-dimensional Euclidean space with vector norm $|\cdot|, \mathbb{R}^{n \times m}$ is the set of all $n \times m$ real matrices. The notation $P \succ 0$, for $P \in \mathbb{R}^{n \times n}$, means that $P$ is symmetric and positive definite. The set $\mathcal{S}_{n}^{+}$represents the set of symmetric positive definite matrices of $\mathbb{R}^{n \times n}$. The set of continuous functions from an interval $\mathcal{I} \subset \mathbb{R}$ to $\mathbb{R}^{n}$ which are square integrable is denoted as space $\mathcal{L}_{2}\left(\mathcal{I} \rightarrow \mathbb{R}^{n}\right)$. The symmetric matrix $\left[\begin{array}{ll}A & B \\ * & C\end{array}\right]$ stands for $\left[\begin{array}{cc}A & B \\ B^{T} & C\end{array}\right]$ and $\operatorname{diag}(A, B)$ stands for the diagonal matrix $\left[\begin{array}{cc}A & 0 \\ 0 & B\end{array}\right]$. Moreover, for any square matrix $A \in \mathbb{R}^{n \times n}$, we define $\operatorname{He}(A)=A+A^{T}$. The matrix $I$ represents the identity matrix of appropriate dimension. The notation $0_{n, m}$ stands for the matrix in $\mathbb{R}^{n \times m}$ whose entries are zero and, when no confusion is possible, the subscript will be omitted. The notation $\left(\begin{array}{c}k \\ l\end{array}\right)$ refers to the binomial coefficients given by $\frac{k !}{(k-l) ! l !}$.

\section{Problem formulation}

\subsection{Definition of the system}

Consider a linear time-delay system of the form:

$$
\left\{\begin{array}{l}
\dot{x}(t)=A x(t)+A_{d} \int_{-h}^{0} f(\theta) x(t+\theta) d \theta, \forall t \geq 0 \\
x(t)=\phi(t), \quad \forall t \in[-h, 0]
\end{array}\right.
$$

where $x(t) \in \mathbb{R}^{n}$ is the state vector, $\phi$ is a continuous function, representing the initial conditions, $A$ and $A_{d}$ are constant matrices and $f$ denotes a known continuous function of $\mathcal{L}_{2}([-h, 0] \rightarrow \mathbb{R})$ and represents the kernel of the distributed delay. The delay $h$ is assumed to be constant. The goal of this paper is to provide a generic analysis which is able to assess stability of system (1) for any continuous scalar kernel $f$ and to derive sufficient stability conditions. This paper exploits the properties of the Legendre polynomials to provide new stability conditions for systems with distributed delay. Let us first recall the definition of the Legendre polynomials and their basic properties.

\subsection{Legendre polynomials and kernel approximation}

Let us define the Legendre polynomials considered over the interval $[-h, 0]$

$$
\forall k \in \mathbb{N}, \quad L_{k}(u)=(-1)^{k} \sum_{l=0}^{k} p_{l}^{k}\left(\frac{u+h}{h}\right)^{l},
$$

with $p_{l}^{k}=(-1)^{l}\left(\begin{array}{c}k \\ l\end{array}\right)\left(\begin{array}{c}k+l \\ l\end{array}\right)$. The main idea for employing these polynomials comes from their nice properties that are summarized below.

Property 1 The Legendre polynomials described in (2) represent an orthogonal sequence with respect to the inner product $\int_{-h}^{0} \phi(s) \psi(s) \mathrm{d} s$, for any $\phi, \psi$ in $\mathcal{L}_{2}([-h, 0] \rightarrow \mathbb{R})$ and verify for all $(k, l)$ in $\mathbb{N}^{2}$

$$
\int_{-h}^{0} L_{k}(u) L_{l}(u) \mathrm{d} u= \begin{cases}0, & k \neq l \\ \frac{h}{2 k+1}, & k=l .\end{cases}
$$

The proof can be found in [6]. In light of this property, we will consider the polynomial approximation of the kernel $f$ in $\mathcal{L}_{2}([-h, 0] \rightarrow \mathbb{R})$. For a given integer $N, f$ is rewritten as

$$
\forall \theta \in[-h, 0], \quad f(\theta)=\sum_{k=0}^{N} \frac{2 k+1}{h} f_{k} L_{k}(\theta)+r_{N}(f, \theta),
$$

where

$$
\begin{aligned}
f_{k} & =\int_{-h}^{0} f(u) L_{k}(u) \mathrm{d} u, \quad \forall k=1, \ldots, N, \\
r_{N}(f, \theta) & =f(\theta)-\sum_{k=0}^{N} \frac{2 k+1}{h} f_{k} L_{k}(\theta) .
\end{aligned}
$$

Since Legendre polynomials are orthogonal with respect to the inner product under consideration, this manipulation can be interpreted as the Graam-Schmidt normalization of the function $f$ with respect to the $N$ first Legendre polynomials. Indeed, it can be easily verified that the resulting function $r_{N}$ is orthogonal to the $N$ first Legendre polynomials and represents the remainder of the approximation.

Remark 1 If the kernel $f$ is a polynomial function of degree $N^{*}$, then $r_{N^{*}+1}$ is the null function.

Define $\rho_{N}$ as the square of norm of $r_{N}$ associated with the inner product, i.e.

$$
\rho_{N}(f)=\int_{-h}^{0} r_{N}^{2}(f, \theta) \mathrm{d} \theta
$$

The next lemma provides some properties of $\rho_{N}(f)$. 
Lemma 1 The following inequalities hold for any $f$ in $\mathcal{L}_{2}([-h, 0] \rightarrow \mathbb{R})$ and for any integer $N \geq 0$

$$
\begin{gathered}
\rho_{N}(f)=\int_{-h}^{0} f^{2}(\theta) \mathrm{d} \theta-\frac{1}{h} \sum_{k=0}^{N}(2 k+1) f_{k}^{2} \geq 0, \\
\rho_{N+1}(f)-\rho_{N}(f)=-\frac{2 N+3}{h} f_{N+1}^{2} \leq 0 .
\end{gathered}
$$

Proof: The positivity of $\rho_{N}$ results from its definition. Equation (7) is obtained from the properties of the Legendre polynomials. The proof of (8) is a direct extension of $(7)$.

\subsection{Modelling of distributed delay systems}

Exploiting the polynomial approximation of the kernel $f$, the time-delay system (1) can be rewritten as

$$
\begin{aligned}
\dot{x}(t)= & A x(t)+A_{d} \sum_{k=0}^{N}(2 k+1) f_{k} \Omega_{k}\left(x_{t}\right) \\
& +\rho_{N}(f) A_{d} \Theta_{N}\left(f, x_{t}\right),
\end{aligned}
$$

where

$$
\begin{aligned}
& \Omega_{k}\left(x_{t}\right)=\frac{1}{h} \int_{-h}^{0} L_{k}(\theta) x_{t}(\theta) \mathrm{d} \theta, \\
& \Theta_{N}\left(f, x_{t}\right)= \begin{cases}0, & \text { if } \quad \rho_{N}(f)=0, \\
\frac{1}{\rho_{N}(f)} \int_{-h}^{0} r_{N}(f, \theta) x_{t}(\theta) \mathrm{d} \theta, & \text { if } \quad \rho_{N}(f)>0 .\end{cases}
\end{aligned}
$$

The objective is to provide a stability analysis able to take into account these additional signals.

\section{Bessel-Legendre inequality}

Before stating the main result of the paper, we provide some additional properties of the Legendre polynomials that will be helpful in the remainder of the paper.

Property 2 The Legendre polynomials described in (2) satisfy the following boundary conditions

$$
\forall k \in \mathbb{N}, \quad L_{k}(0)=1, \quad L_{k}(-h)=(-1)^{k} .
$$

Property 3 The Legendre polynomials described in Definition 2 satisfy the following differentiation rule:

$$
\frac{\mathrm{d}}{\mathrm{d} u} L_{k}(u)=\left\{\begin{array}{l}
0, \quad \text { if } k=0, \\
\sum_{i=0}^{k-1} \frac{(2 i+1)}{h}\left(1-(-1)^{k+i}\right) L_{i}(u), \\
\text { if } k \geq 1
\end{array}\right.
$$

Proofs of these properties can be found in [6] and are thus omitted. Based on the Legendre polynomials and an application of Bessel's inequality [6], we obtain the following lemma.

Lemma 2 Let $x \in \mathcal{L}_{2}\left([-h, 0] \rightarrow \mathbb{R}^{n}\right)$, $f$ be a continuous function in $\mathcal{L}_{2}([-h, 0] \rightarrow \mathbb{R})$ and $R \in \mathcal{S}_{n}^{+}$. The integral inequality

$$
\begin{aligned}
\int_{-h}^{0} x^{T}(u) R x(u) \mathrm{d} u \geq & h \sum_{k=0}^{N}(2 k+1) \Omega_{k}^{T}(x) R \Omega_{k}(x) \\
& +\sigma\left(\rho_{N}(f)\right) \Theta_{N}^{T}(f, x) R \Theta_{N}(f, x)
\end{aligned}
$$

holds, for all $N \in \mathbb{N}$, where $\rho_{N}, \Omega_{k}$ and $\Theta_{N}$ have been defined in (6) and (10) respectively, and where

$$
\sigma\left(\rho_{N}(f)\right)=\left\{\begin{array}{lll}
\rho_{N}(f) & \text { if } & \rho_{N}(f)>0 \\
1 & \text { if } & \rho_{N}(f)=0
\end{array}\right.
$$

Proof : Consider a function $x$ in $\mathcal{L}_{2}\left([-h, 0] \rightarrow \mathbb{R}^{n}\right), f$ in $\mathcal{L}_{2}([-h, 0] \rightarrow \mathbb{R})$ and a matrix $R$ in $\mathcal{S}_{n}^{+}$. Define the function $z_{N}$ as follows

$z_{N}(u)=x(u)-\sum_{k=0}^{N}(2 k+1) L_{k}(u) \Omega_{k}(x)-r_{N}(f, u) \Theta_{N}(f, x)$.

From its definition, $z_{N}$ is in $\mathcal{L}_{2}\left([-h, 0] \rightarrow \mathbb{R}^{n}\right)$ and the integral $\int_{-h}^{0} z_{N}^{T}(u) R z_{N}(u) \mathrm{d} u$ exists. For the sake of simplicity the arguments of $\rho_{N}, \Omega_{k}$ and $\Theta_{N}$ will be omitted in the following developments. From the orthogonal property of the Legendre polynomials, one has

$$
\begin{aligned}
& \int_{-h}^{0} z_{N}^{T}(u) R z_{N}(u) \mathrm{d} u=\int_{-h}^{0} x^{T}(u) R x(u) \mathrm{d} u \\
& \quad-2 \sum_{k=0}^{N}(2 k+1)\left(\int_{-h}^{0} L_{k}(u) x(u) \mathrm{d} u\right)^{T} R \Omega_{k} \\
& \quad+\sum_{k=0}^{N}(2 k+1)^{2}\left(\int_{-h}^{0} L_{k}^{2}(u) \mathrm{d} u\right) \Omega_{k}^{T} R \Omega_{k} \\
& \quad+\left(\int_{-h}^{0} r_{N}^{2}(f, u) \mathrm{d} u\right) \Theta_{N}^{T} R \Theta_{N} \\
& \quad-2\left(\int_{-h}^{0} r_{N}(f, u) x(u) \mathrm{d} u\right)^{T} R \Theta_{N} \\
& \quad+2 \sum_{k=0}^{N}(2 k+1)\left(\int_{-h}^{0} r_{N}(f, u) L_{k}(u) \mathrm{d} u\right) \Omega_{k}^{T} R \Theta_{N} .
\end{aligned}
$$

On the one hand, assume first that $\rho_{N}$, and consequently $r_{N}$, are not zero. From the definition of $\Omega_{k}$ and $\Theta_{N}$ and noting that $(2 k+1)^{2} \int_{-h}^{0} L_{k}^{2}(u) d u=(2 k+1) h$, it yields

$$
\begin{aligned}
& \int_{-h}^{0} z_{N}^{T}(u) R z_{N}(u) \mathrm{d} u=\int_{-h}^{0} x^{T}(u) R x(u) \mathrm{d} u \\
& \quad-\sum_{k=0}^{N}(2 k+1) h \Omega_{k}^{T} R \Omega_{k}-\rho_{N} \Theta_{N}^{T} R \Theta_{N} \\
& \quad+2 \sum_{k=0}^{N} \frac{2 k+1}{h}\left(\int_{-h}^{0} r_{N}(f, u) L_{k}(u) \mathrm{d} u\right) \Omega_{k}^{T} R \Theta_{N} .
\end{aligned}
$$


Moreover, for any $k=0, \ldots, N$, one has

$$
\begin{aligned}
\int_{-h}^{0} r_{N}(f, u) L_{k}(u) \mathrm{d} u & =\int_{-h}^{0} f(u) L_{k}(u) \mathrm{d} u \\
& -\sum_{i=0}^{N} \frac{2 i+1}{h} f_{i} \int_{-h}^{0} L_{i}(u) L_{k}(u) \mathrm{d} u
\end{aligned}
$$

From the Property 1, and the definition of $r_{N}$, we show that $\int_{-h}^{0} r_{N}(f, u) L_{k}(u) \mathrm{d} u=0$. It thus follows that

$$
\begin{aligned}
\int_{-h}^{0} z_{N}^{T}(u) R z_{N}(u) \mathrm{d} u & =\int_{-h}^{0} x^{T}(u) R x(u) d u \\
& -\sum_{k=0}^{N}(2 k+1) h \Omega_{k}^{T} R \Omega_{k}-\rho_{N} \Theta_{N}^{T} R \Theta_{N},
\end{aligned}
$$

which concludes the proof for the case $\rho_{n} \neq 0$. On the other hand, if $\rho_{N}=0$ (and $r_{N}=0$ ), the following equation holds

$$
\begin{aligned}
\int_{-h}^{0} z_{N}^{T}(u) R z_{N}(u) \mathrm{d} u & =\int_{-h}^{0} x^{T}(u) R x(u) \mathrm{d} u \\
& -\sum_{k=0}^{N}(2 k+1) h \Omega_{k}^{T} R \Omega_{k}-\Theta_{N}^{T} R \Theta_{N}
\end{aligned}
$$

because $\Theta_{N}\left(f, x_{t}\right)=0$. The result is finally obtained by combining the two previous equations and noting that the left hand side of the previous equations is positive definite.

In the remainder of the paper, the following corollary of the previous lemma will be used. Note that it also corresponds to an inequality already presented in [20,21].

Corollary 3 Let $x \in \mathcal{L}_{2}\left([-h, 0] \rightarrow \mathbb{R}^{n}\right)$ and $R \in \mathcal{S}_{n}^{+}$. The integral inequality

$$
\int_{-h}^{0} x^{T}(u) R x(u) \mathrm{d} u \geq h \sum_{k=0}^{N}(2 k+1) \Omega_{k}^{T} R \Omega_{k}
$$

holds, for all $N \in \mathbb{N}$, where $\Omega_{k}, k=0,1 \ldots, N$ have been defined in Lemma 2.

Proof: The proof is obtained by removing the term $\rho_{N}(f) \Theta_{N}^{T}(x) R \Theta_{N}(x)$ from (11).

Remark 2 The previous inequality encompasses the Jensen inequality [9] and the recent Wirtinger-based integral inequality [19] as particular cases for $N=0$ and $N=1$, respectively. Thus, the set of inequalities provided in Corollary 3 represents a more general formulation than these two inequalities. Additionally, the Parseval identity proves that inequality (14) becomes asymptotically non conservative as $N$ goes to infinity.

\section{Application to stability analysis}

\subsection{Selection of Lyapunov-Krasovskii functionals}

Consider the functional given by

$$
\begin{aligned}
V\left(x_{t}\right)= & x^{T}(t) P x(t)+2 x^{T}(t) \int_{-h}^{0} Q(\theta) x_{t}(\theta) \mathrm{d} \theta \\
& +\int_{-h}^{0} x_{t}^{T}(\theta) S(\theta) x_{t}(\theta) \mathrm{d} \theta \\
& +\int_{-h}^{0} \int_{-h}^{0} x_{t}\left(\theta_{1}\right) T\left(\theta_{1}, \theta_{2}\right) x_{t}\left(\theta_{2}\right) \mathrm{d} \theta_{1} \mathrm{~d} \theta_{2},
\end{aligned}
$$

where $x_{t}(\theta)=x(t+\theta)$ represents the state of the timedelay system and $h>0$ the delay and where the matrix $P$ is symmetric positive definite and the functions $Q, S$ and $T$ are differentiable (see Section 7.4 in [9] for more details). Theorem 7.3 from [9] ensures that if a time-delay system is asymptotically stable, then $V$ is a Lyapunov-Krasovskii functional, provided that $Q$ and $T$ satisfy some specific partial differential equations. In practice, numerically checking the existence of such functionals often requires an approximation of $Q, S$ and $T$. In [9] a discretization method was proposed where the functions $Q, T$ and $S$ were chosen piecewise linear and the conditions are presented through the LMI setup. In [17], these matrices were chosen as polynomial functions and the numerical test was performed using the SOSTOOLS. Here, we aim at choosing the structure of the Lyapunov Krasovskii functional based on the use of Legendre polynomials. Considering the functional (15), we propose to model the different matrices $P, Q(\theta), T(\theta, s)$ and $S(\theta)$ as polynomials with respect to the variables $\theta$ and $s$. Contrary to a Sum of Squares (SOS) formulation [17], these polynomials are expressed in terms of the Legendre basis as follows:

$$
Q(\theta)=\sum_{i=0}^{N} Q_{i} L_{i}(\theta), T\left(\theta_{1}, \theta_{2}\right)=\sum_{i=0}^{N} \sum_{j=0}^{N} L_{i}\left(\theta_{1}\right) L_{j}\left(\theta_{2}\right) T_{i j},
$$

where the matrices $Q_{i}, T_{i j}=T_{j i}^{T}$, for $i, j=0, \ldots, N$ have to be optimized. The polynomial matrix $S(\theta)$ is chosen as a linear function with respect to $\theta$ and is therefore simply expressed with the canonical basis $S(\theta)=S+(h+\theta) R$. This selection is consistent with the complete LyapunovKrasovskii functional defined in [9] (see section 5.6.3, p. 181). Hence, we can define the functional $V_{N}$ (the subscript $N$ denoting the order of the polynomial approximation) as follows :

$$
\begin{aligned}
V_{N}\left(x_{t}\right)= & \zeta_{N}^{T}\left(x_{t}\right) P_{N} \zeta_{N}\left(x_{t}\right)+\int_{-h}^{0} x_{t}^{T}(\theta) S x_{t}(\theta) \mathrm{d} \theta \\
& +\int_{-h}^{0}(h+\theta) x_{t}^{T}(\theta) R x_{t}(\theta) \mathrm{d} \theta
\end{aligned}
$$


where $P_{N}=\left[\begin{array}{cccc}P & Q_{0} & \ldots & Q_{N} \\ Q_{0}^{T} & T_{00} & \ldots & T_{0 N} \\ \vdots & \vdots & & \vdots \\ Q_{N}^{T} & T_{N 0} & \ldots & T_{N N}\end{array}\right]$ and

$$
\zeta_{N}\left(x_{t}\right)=\left[\begin{array}{c}
x_{t}(0) \\
\int_{-h}^{0} L_{0}(\theta) x_{t}(\theta) \mathrm{d} \theta \\
\vdots \\
\int_{-h}^{0} L_{N}(\theta) x_{t}(\theta) \mathrm{d} \theta
\end{array}\right]=\left[\begin{array}{c}
x_{t}(0) \\
h \Omega_{0}\left(x_{t}\right) \\
\vdots \\
h \Omega_{N}\left(x_{t}\right)
\end{array}\right], N \geq 0
$$

collects the current state and the projections of the state function $x_{t}$ onto the $N$ first Legendre polynomials.

\subsection{Stability analysis}

We provide here a first stability result for distributed delay systems, which is based on the proposed Lyapunov Krasovskii functional (16) and the use of the inequality provided in Lemma 2.

Theorem 4 Consider system (1) with a continuous scalar kernel $f$ in $\mathcal{L}_{2}([-h, 0] \rightarrow \mathbb{R})$ and with a given $h>0$. If, for a given integer $N \geq 0$, there exist a matrix $P_{N} \in \mathcal{S}_{(N+2) n}^{+}$, and two matrices $S, R \in \mathcal{S}_{n}^{+}$such that the following LMIs

$$
\begin{gathered}
\Phi_{N}^{+}(h):=P_{N}+\frac{1}{h} S_{N} \succ 0 \\
\Phi_{N}^{-}\left(h, \rho_{N}(f)\right)=\left[\begin{array}{cc}
\Phi_{N 0}(h)-h R_{N} & \rho_{N}(f) \Phi_{N 1}(h) \\
\rho_{N}(f) \Phi_{N 1}^{T}(h) & -\sigma\left(\rho_{N}(f)\right) R
\end{array}\right] \prec 0
\end{gathered}
$$

are satisfied where the function $\sigma$ is defined in Lemma 2 and where

$$
\begin{aligned}
& \Phi_{N 0}(h)=\operatorname{He}\left(G_{N}^{T}(h) P_{N} H_{N}(h)\right)+\Sigma_{N}(h), \\
& \Phi_{N 1}(h)=G_{N}^{T}(h) P_{N} J_{N} A_{d}, \\
& \Sigma_{N}(h)=\operatorname{diag}\left(S+h R,-S, 0_{(N+1) n}\right) \text {, } \\
& S_{N} \quad=\operatorname{diag}(0, S, 3 S, \ldots,(2 N+1) S), \\
& R_{N} \quad=\operatorname{diag}(0,0, R, 3 R, \ldots,(2 N+1) R) \text {, } \\
& G_{N}(h)=\left[\begin{array}{ccc}
I & 0_{n} & 0_{n, n(N+1)} \\
0_{n(N+1), n} & 0_{n(N+1), n} & h I_{n(N+1)}
\end{array}\right], \\
& H_{N}=\left[\begin{array}{llll}
F_{N}^{T} & \Gamma_{N}^{T}(0) & \Gamma_{N}^{T}(1) \ldots \Gamma_{N}^{T}(N)
\end{array}\right]^{T}, \\
& F_{N}=\left[\begin{array}{llllll}
A & 0 & f_{0} A_{d} & 3 f_{1} A_{d} & \ldots & (2 N+1) f_{N} A_{d}
\end{array}\right] \text {, } \\
& J_{N} \quad=\left[\begin{array}{ll}
I & 0_{n, n(N+1)}
\end{array}\right]^{T} \text {, } \\
& \Gamma_{N}(k)=\left[I(-1)^{k+1} I \gamma_{N k}^{0} I \ldots \gamma_{N k}^{N} I\right] \text {, } \\
& \gamma_{N k}^{i}= \begin{cases}-(2 i+1)\left(1-(-1)^{k+i}\right), & \text { if } \quad i \leq k, \\
0, & \text { if } \quad i \geq k+1,\end{cases}
\end{aligned}
$$

then, the distributed delay system (1) is asymptotically stable for the constant delay $h$.

Proof : Consider the Lyapunov Krasovskii functional (16). Following the procedure provided in [9], Corollary 3 can be applied to the second term of $V_{N}$ since $S \succ 0$ to obtain a lower bound of $V_{N}$. In order to be consistent with the definition of $\zeta_{N}$, Corollary 3 is considered with the order $N$. It thus yields

$$
\begin{aligned}
\int_{-h}^{0} x_{t}^{T}(s) S x_{t}(s) \mathrm{d} s & \geq h \sum_{k=0}^{N}(2 k+1) \Omega_{k}^{T}\left(x_{t}\right) S \Omega_{k}\left(x_{t}\right) \\
& =\frac{1}{h} \zeta_{N}^{T}\left(x_{t}\right) S_{N} \zeta_{N}\left(x_{t}\right) .
\end{aligned}
$$

Hence, it holds

$$
\begin{aligned}
V_{N}\left(x_{t}\right) \geq & \zeta_{N}^{T}\left(x_{t}\right) \Phi_{N}^{+}(h) \zeta_{N}\left(x_{t}\right) \\
& +\int_{-h}^{0}(h+s) x_{t}^{T}(s) R x_{t}(s) \mathrm{d} s
\end{aligned}
$$

Consequently, if the matrices $R$ and $\Phi_{N}^{+}(h)$ are positive definite, there exists a positive scalar $\epsilon_{1}>0$ such that $V_{N}\left(x_{t}\right) \geq \epsilon_{1}\left|x_{t}(0)\right|^{2}$. Furthermore, there always exists a sufficiently large scalar $\lambda>0$ such that $P_{N} \preceq$ $\lambda \operatorname{diag}(I, I, 3 I, \ldots,(2 N+1) I)$, yielding

$$
\begin{aligned}
V_{N}\left(x_{t}\right) \leq & \lambda\left|x_{t}(0)\right|^{2}+\lambda \sum_{i=0}^{N-1}(2 i+1) \Omega_{i}^{T}\left(x_{t}\right) \Omega_{i}\left(x_{t}\right) \\
& +\int_{-h}^{0} x_{t}^{T}(s)(S+h R) x_{t}(s) \mathrm{d} s .
\end{aligned}
$$

Applying Corollary 3 to the second term of the righthand side ensures that

$V_{N}\left(x_{t}\right) \leq \lambda\left|x_{t}(0)\right|^{2}+\int_{-h}^{0} x_{t}^{T}(s)(\lambda h I+S+h R) x_{t}(s) \mathrm{d} s$

which guarantees that there exists a scalar $\epsilon_{2}>0$, such that $V_{N}\left(x_{t}\right) \leq \epsilon_{2}\left|x_{t}\right|_{h}^{2}$, where the notation $\left|x_{t}\right|_{h}$ stands for $\left.\sup _{\theta \in[-h} 0\right]\left|x_{t}(\theta)\right|$. Then it holds

$$
\epsilon_{1}\left|x_{t}(0)\right|^{2} \leq V_{N}\left(x_{t}\right) \leq \epsilon_{2}\left|x_{t}\right|_{h}^{2}
$$

The computation of $\dot{V}_{N}$ leads to

$$
\begin{aligned}
\dot{V}_{N}\left(x_{t}\right)= & 2 \zeta_{N}^{T}\left(x_{t}\right) P_{N} \dot{\zeta}_{N}\left(x_{t}\right)+x_{t}^{T}(0)(S+h R) x_{t}(0) \\
& -x_{t}^{T}(-h) S x_{t}(-h)-\int_{-h}^{0} x_{t}^{T}(s) R x_{t}(s) \mathrm{d} s
\end{aligned}
$$

We aim at finding an upper bound of the functional $\dot{V}_{N}$ using the vector $\Theta_{N}\left(f, x_{t}\right)$ and the augmented vector $\xi_{N}$ given, for any integer $N$, by

$\xi_{N}\left(x_{t}\right)=\left[\begin{array}{llll}x_{t}^{T}(0) & x_{t}^{T}(-h) \Omega_{0}^{T}\left(x_{t}\right) \Omega_{1}^{T}\left(x_{t}\right) \ldots \Omega_{N}^{T}\left(x_{t}\right)\end{array}\right]^{T}$ 
Consider the first term of the right hand side of (20). It is easy to see that

$$
\dot{\zeta}_{N}\left(x_{t}\right)=\left[\begin{array}{c}
\dot{x}_{t}(0) \\
h \dot{\Omega}_{0}\left(x_{t}\right) \\
\vdots \\
h \dot{\Omega}_{N}\left(x_{t}\right)
\end{array}\right]=\left[\begin{array}{c}
\dot{x}_{t}(0) \\
h \Omega_{0}\left(\dot{x}_{t}\right) \\
\vdots \\
h \Omega_{N}\left(\dot{x}_{t}\right)
\end{array}\right] .
$$

The definition of $F_{N}$ and the equation (9) yield

$$
\dot{x}_{t}(0)=F_{N} \xi_{N}\left(x_{t}\right)+\rho_{N}(f) A_{d} \Theta_{N}\left(f, x_{t}\right) .
$$

Then, an integration by parts ensures that

$$
\begin{aligned}
\forall k \leq N, \quad h \Omega_{k}\left(\dot{x}_{t}\right)= & L_{k}(0) x_{t}(0)-L_{k}(-h) x_{t}(-h) \\
& -\int_{-h}^{0} \dot{L}_{k}(u) x_{t}(u) \mathrm{d} u
\end{aligned}
$$

Thanks to Properties 2 and 3 of the Legendre polynomials, the following expression is derived

$$
\begin{aligned}
h \Omega_{k}\left(\dot{x}_{t}\right) & =x_{t}(0)-(-1)^{k} x_{t}(-h)-\sum_{i=0}^{k-1} \gamma_{N k}^{i} \Omega_{i}\left(x_{t}\right) \\
& =\Gamma_{N}(k) \xi_{N}\left(x_{t}\right) .
\end{aligned}
$$

Gathering all the components of $\dot{\zeta}_{N}(t)$, we obtain

$$
\dot{\zeta}_{N}\left(x_{t}\right)=H_{N} \xi_{N}\left(x_{t}\right)+\rho_{N}(f) J_{N} A_{d} \Theta_{N}\left(f, x_{t}\right),
$$

where the matrices $H_{N}$ and $J_{N}$ are given in the statement of the theorem. Since $\zeta_{N}\left(x_{t}\right)=G_{N}(h) \xi_{N}\left(x_{t}\right)$ and $x_{t}^{T}(0)(S+h R) x_{t}(0)-x_{t}^{T}(-h) S x_{t}(-h)=\xi_{N}^{T}\left(x_{t}\right) \Sigma_{N}(h)$ $\xi_{N}\left(x_{t}\right)$, we have

$$
\begin{aligned}
\dot{V}_{N}\left(x_{t}, \dot{x}_{t}\right)= & \bar{\xi}_{N}^{T}\left[\begin{array}{cc}
\Phi_{N 0}(h) & \rho_{N}(f) \Phi_{N 1}(h) \\
\rho_{N}(f) \Phi_{N 1}^{T}(h) & 0
\end{array}\right] \bar{\xi}_{N}\left(x_{t}\right) \\
& -\int_{-h}^{0} x_{t}^{T}(s) R x_{t}(s) \mathrm{d} s .
\end{aligned}
$$

where $\bar{\xi}_{N}\left(x_{t}\right)=\left[\xi_{N}^{T}\left(x_{t}\right) \Theta_{N}^{T}\left(f, x_{t}\right)\right]^{T}$. Applying Lemma 2 to the order $N$, we obtain

$$
\dot{V}_{N}\left(x_{t}\right) \leq \bar{\xi}_{N}^{T}\left(x_{t}\right) \Phi_{N}^{-}\left(h, \rho_{N}(f)\right) \bar{\xi}_{N}\left(x_{t}\right) .
$$

Therefore, if the matrix $\Phi_{N}^{-}\left(h, \rho_{N}(f)\right)$ is negative definite, there exists a positive scalar $\epsilon_{3}$ such that

$$
\dot{V}_{N}\left(x_{t}\right) \leq-\epsilon_{3}\left|x_{t}(0)\right|^{2} .
$$

To conclude, if the LMI conditions (17) and (18) are satisfied, the functional meets the requirements of the Lyapunov-Krasovskii theorem, (19) and (23), and the distributed delay system (1) is asymptotically stable for the constant delay $h$.
Remark 3 A notable aspect of this theorem is the possibility to extend it to robust stability where the matrices $A$ and $A_{d}$ are uncertain but belong to a given polytope since the $\Psi^{-}(h)$ is affine with respect to these matrices.

Remark 4 In the stability conditions derived in Theorem 4 , the integer $N$ appears as a tuning parameter of the LMIs. As we will show in Section 5, increasing $N$ may lead to a reduction of the conservatism, of course at the price of increasing of the computational cost. Indeed the number of decision variables and the size of the LMIs also increase with $N$.

\subsection{Distributed delay systems with two kernels}

Theorem 7 only addresses the case of scalar kernels $f$. Extensions to matrix kernels corresponding to the following system

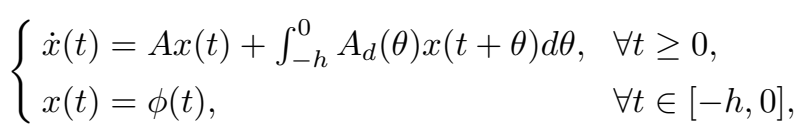

is not direct since the methodology developed in Theorem 7 relies on the use of the term $\int_{-h}^{0} f(\theta) x_{t}(\theta) \mathrm{d} \theta$. This limitation can be easily undertaken by splitting the matrix kernel $A_{d}(\theta)$ into several pieces which can be treated following the first theorem. In this subsection, we present the simplest extension which consists of the following distributed delay systems with two distributed terms:

$$
\left\{\begin{aligned}
& \dot{x}(t)=A x(t)+ \int_{-h}^{0}\left[f(\theta) A_{d f}+g(\theta) A_{d g}\right] x(t+\theta) \mathrm{d} \theta \\
& \forall t \geq 0 \\
& x(t)=\phi(t), \quad \forall t \in[-h, 0]
\end{aligned}\right.
$$

where $x(t) \in \mathbb{R}^{n}$ is the state vector, $\phi$ is a continuous initial condition function, $A, A_{d f}$ and $A_{d g}$ are constant matrices and $f, g$ are two known continuous functions over the interval $[-h, 0]$. The delay $h$ is assumed to be constant. Following the procedure as in the simple kernel case, system (24) is rewritten as

$$
\left\{\begin{aligned}
\dot{x}(t)= & A x(t)+\sum_{k=0}^{N}(2 k+1) A_{d}^{k} \Omega_{k}\left(x_{t}\right) & & \\
& +\left[\rho_{N}(f) A_{d f} \rho_{N}(g) A_{d g}\right] \bar{\Theta}_{N}\left(x_{t}\right), & & \forall t \geq 0, \\
x(t)= & \phi(t), & & \forall t \in[-h, 0],
\end{aligned}\right.
$$

where $A_{d}^{k}=f_{k} A_{d f}+g_{k} A_{d g}$ and

$$
g_{k}=\int_{-h}^{0} g(\theta) L_{k}(\theta) \mathrm{d} \theta, \quad \bar{\Theta}_{N}\left(x_{t}\right)=\left[\begin{array}{c}
\Theta_{N}(f, \theta) \\
\Theta_{N}(g, \theta)
\end{array}\right] .
$$

The following lemma and theorem are adapted from Section 4.2 . 
Lemma 5 Let $x \in \mathcal{L}_{2}\left([-h, 0] \rightarrow \mathbb{R}^{n}\right), f$ and $g$ be two continuous functions in $[-h, 0] \rightarrow \mathbb{R}^{n}$ and $R \in \mathcal{S}_{n}^{+}$. The integral inequality

$$
\begin{aligned}
\int_{-h}^{0} x(u) R x(u) \mathrm{d} u & \geq h \sum_{k=0}^{N}(2 k+1) \Omega_{k}^{T}(x) R \Omega_{k}(x) \\
+ & \bar{\Theta}_{N}^{T}(x)\left[\begin{array}{cc}
\sigma\left(\rho_{N}(f)\right) R & \beta_{N}(f, g) R \\
\beta_{N}(f, g) R & \sigma\left(\rho_{N}(g)\right) R
\end{array}\right] \bar{\Theta}_{N}(x),
\end{aligned}
$$

holds, for all $N \in \mathbb{N}$, where

$$
\beta_{N}(f, g)=\rho_{N}(f) \rho_{N}(g) \int_{-h}^{0} r_{N}(f, \theta) r_{N}(g, \theta) \mathrm{d} \theta
$$

and where $\Omega_{k}, \bar{\Theta}_{N}, \rho_{N}$ and the function $\sigma$ have been defined earlier.

Theorem 6 For a given integer $N \geq 0$ and a constant delay $h$, assume that there exist a matrix $P_{N} \in \mathcal{S}_{(N+2) n}^{+}$, and two matrices $S, R \in \mathcal{S}_{n}^{+}$such that $\Phi_{N}^{+}(h) \succ 0$ and

$$
\Phi_{N}^{+}(h):=P_{N}+\frac{1}{h} S_{N} \succ 0,
$$

where

$$
\begin{aligned}
& \Phi_{N}^{-}\left(h, \rho_{N}\right)= \\
& {\left[\begin{array}{ccc}
\bar{\Phi}_{N 0}(h)-h R_{N} & \rho_{N}(f) \Phi_{N 1}(h) & \rho_{N}(g) \Phi_{N 2}(h) \\
\rho_{N} \Phi_{N 1}^{T}(h) & -\sigma\left(\rho_{N}(f)\right) R & -\beta_{N}(f, g) R \\
\gamma_{N} \Phi_{N 2}^{T}(h) & -\beta_{N}(f, g) R & -\sigma\left(\rho_{N}(g)\right) R
\end{array}\right] \prec 0,} \\
& \bar{\Phi}_{N 0}(h)=\operatorname{He}\left(G_{N}^{T}(h) P_{N} \bar{H}_{N}\right)+\Sigma_{N}(h), \\
& \Phi_{N 1}(h)=G_{N}^{T}(h) P_{N} J_{N} A_{d f}, \\
& \Phi_{N 2}(h)=G_{N}^{T}(h) P_{N} J_{N} A_{d g}, \\
& \bar{H}_{N}=\left[\bar{F}_{N}^{T} \Gamma_{N}^{T}(0) \Gamma_{N}^{T}(1) \ldots \Gamma_{N}^{T}(N)\right]^{T}, \\
& \bar{F}_{N}=\left[\begin{array}{llllll}
A & 0 & A_{d}^{1} & 3 A_{d}^{2} & \ldots & (2 N+1) A_{d}^{N}
\end{array}\right],
\end{aligned}
$$

with $\Sigma_{N}(h), S_{N}, R_{N}, J_{N}, \Gamma_{N}(k)$ and $G_{N}(h)$ defined in Theorem 4. Then system (24) is asymptotically stable for the constant delay $h$.

\subsection{Comments on systems with a single discrete delay.}

By a slight modification, the stability conditions from Theorem 4 can be extended to the case of linear systems with a pointwise delay, defined by

$$
\begin{cases}\dot{x}(t)=A x(t)+A_{d} x(t-h), & \forall t \geq 0, \\ x(t)=\phi(t), & \forall t \in[-h, 0],\end{cases}
$$

where $A, A_{d}, h$ and $\phi$ satisfy the same assumptions of Theorem 4 . Following the same procedure as previously, the next theorem is stated.
Theorem 7 For a given integer $N \geq 0$ and a constant delay $h$, assume that there exist a matrix $P_{N} \in \mathcal{S}_{(N+2) n}^{+}$, and two matrices $S, R \in \mathcal{S}_{n}^{+}$such that $\Phi_{N}^{+}(h) \succ 0$ and

$$
\Psi_{N}^{-}(h)=\Psi_{N 0}(h)-h R_{N} \prec 0,
$$

are satisfied where

$$
\begin{aligned}
\Psi_{N 0}(h) & =\operatorname{He}\left(G_{N}^{T}(h) P \tilde{H}_{N}\right)+\Sigma_{N}(h), \\
\tilde{H}_{N} & =\left[\begin{array}{ccccc}
\tilde{F}_{N}^{T} & \Gamma_{N}^{T}(0) & \Gamma_{N}^{T}(1) & \ldots & \Gamma_{N}^{T}(N)
\end{array}\right]^{T}, \\
\tilde{F}_{N} & =\left[\begin{array}{llllll}
A & A_{d} & 0 & 0 & \ldots & 0
\end{array}\right],
\end{aligned}
$$

with $\Phi_{N}^{+}(h), R_{N}, G_{N}(h), \Sigma_{N}(h)$ and $\Gamma_{N}(i), i=0, \ldots, N$ defined in Theorem 4. Then system (29) is asymptotically stable for the constant delay $h$.

Proof: The proof strictly follows the one of Theorem 4 except for equation (21), which is replaced by $\dot{x}(t)=\tilde{F}_{N} \xi_{N}(t)$.

Remark 5 The LMI of Theorem 7 are equivalent to the stability condition provided in [21], which only copes with pointwise delays. In [20], another class of LyapunovKrasovskii functionals was employed which contains a double integral term in $\dot{x}(t)$.

\section{$5 \quad$ Hierarchy of LMI stability conditions}

This section aims at proving that the previous stability conditions form a hierarchy of LMI conditions. This is formulated in the following theorem based on the stability conditions of Theorem 4 .

Theorem 8 For any time delay system (1), define the set $\mathcal{H}_{N}$ by

$$
\begin{aligned}
& \mathcal{H}_{N}:= \\
& \left\{\begin{array}{c}
h>0: \exists\left(P_{N}, S(N), R(N)\right) \in \mathcal{S}_{(N+2) n} \times\left(\mathcal{S}_{n}^{+}\right)^{2}, \\
\text { s.t. } \Phi_{N}^{+}(h) \succ 0, \Phi_{N}^{-}(h) \prec 0
\end{array}\right\} .
\end{aligned}
$$

Then, the inclusion $\mathcal{H}_{N} \subset \mathcal{H}_{N+1}$ holds for any $N \geq 0$.

Proof : Let $N \in \mathbb{N}$. If $\mathcal{H}_{N}$ is empty, the inclusion is trivial. Assume that $\mathcal{H}_{N}$ is not empty and consider an element $h \in \mathcal{H}_{N}$. From the definition of $\mathcal{H}_{N}$, there exist $P_{N}=P_{N}^{T}, S(N) \succ 0$ and $R(N) \succ 0$ such that $\Phi_{N}^{+}(h) \succ 0$ and $\Phi_{N}^{-}\left(h, \rho_{N}(f)\right) \prec 0$. Taking advantages of the Lyapunov Krasovskii functional (16), we suggest the matrices

$$
P_{N+1}=\left[\begin{array}{cc}
P_{N} & 0 \\
0 & 0
\end{array}\right], \quad \begin{aligned}
& S(N+1)=S(N)=S, \\
& R(N+1)=R(N)=R .
\end{aligned}
$$


Clearly this selection ensures that the functional implies $\Phi_{N+1}^{+}(h) \succ 0$, because for this selection of $P_{N+1}$, we have

$$
\begin{aligned}
\Phi_{N+1}^{+}(h) & =\left[\begin{array}{cc}
P_{N}+\frac{1}{h} S_{N} & 0 \\
0 & \frac{1}{h}(2 N+3) S
\end{array}\right] \\
& =\left[\begin{array}{cc}
\Phi_{N}^{+}(h) & 0 \\
0 & \frac{1}{h}(2 N+3) S
\end{array}\right] .
\end{aligned}
$$

Assume now that $\Phi_{N}^{-}(h) \prec 0$ and $\rho_{N}(f) \neq 0$. Then, by application of the Schur complement it holds

$$
\Phi_{N 0}(h)-h R_{N}+\rho_{N}(f) \Phi_{N 1}(h) R^{-1} \Phi_{N 1}^{T}(h) \prec 0 .
$$

From (8), we get that $\rho_{N}(f)=\rho_{N+1}(f)+\frac{2 N+3}{h} f_{N+1}^{2}$. Reinjecting this relation into the previous equation and applying twice the Schur complement leads to

$$
\begin{aligned}
& \Phi_{N}^{-}\left(h, \rho_{N}(f)\right) \prec 0 \\
& {\left[\begin{array}{ccc}
\Phi_{N 0}(h)-h R_{N} & \Leftrightarrow & \Leftrightarrow \\
(2 N+3) f_{N+1} \Phi_{N 1}^{T}(h) & -(2 N+3) f_{N+1} \Phi_{N 1}(h) & \rho_{N+1} \Phi_{N 1}(h) \\
\rho_{N+1} \Phi_{N 1}^{T}(h) & 0 & 0 \\
& & -\rho_{N+1} R
\end{array}\right] \prec 0 .}
\end{aligned}
$$

According to the construction of the matrices $G_{N}, H_{N}$, $F_{N}$ and $\widetilde{S}_{N}$, some matrix computations show that

$$
\begin{aligned}
& {\left[\begin{array}{cc}
\Phi_{N 0}(h)-h R_{N} & (2 N+3) f_{N+1} \Phi_{N 1}(h) \\
(2 N+3) f_{N+1} \Phi_{N 1}^{T}(h) & -(2 N+3) h R
\end{array}\right]} \\
& =\Phi_{(N+1) 0}(h)-h R_{N+1}, \quad\left[\begin{array}{c}
\Phi_{N 1}(h) \\
0
\end{array}\right]=\Phi_{(N+1) 1}(h),
\end{aligned}
$$

which ensure the following equivalence, under the constraint $P_{N+1}=\left[\begin{array}{cc}P_{N} & 0 \\ 0 & 0\end{array}\right]$

$$
\Phi_{N}^{-}\left(h, \rho_{N}(f)\right) \prec 0 \Leftrightarrow \Phi_{N+1}^{-}\left(h, \rho_{N+1}(f)\right) \prec 0 .
$$

The case $\rho_{N}=0$ follows straightforwardly because in this situation, it must hold $f_{N+1}=0$ and $\rho_{N+1}=0$. Consequently, if there exists a solution to the LMI conditions of Theorem 4 at the order $N$, then there also exists a solution to the LMIs at the order $N+1$ which proves that $h$ belongs to $\mathcal{H}_{N+1}$, which allows to conclude that $\mathcal{H}_{N} \subset \mathcal{H}_{N+1}$.

Since Theorem 4 only provides sufficient stability condition, the sequence of sets $\left\{\mathcal{H}_{N}\right\}_{N \in \mathbb{N}}$ is an increasing sequence of sets representing an inner approximation of the stability pockets. However, the previous theorem does not prove that the conditions of Theorem 4 will converge to the analytical bounds of the delay. Extensions of this previous theorem to Theorems 6 and 7 are similarly derived.

\begin{tabular}{|c|c|c|}
\hline Theorems & $h_{\max }$ & number of variables \\
\hline \hline$[2]$ ("analytical" bound) & 1.498 & - \\
\hline$[24]$ & 1.03 & 3 \\
\hline Th.4 $(N=0)$ & 0.95 & 5 \\
\hline Th.4 $(N=1)$ & 1.45 & 8 \\
\hline Th.4 $(N=2)$ & 1.497 & 12 \\
\hline Th.4 $(N=3)$ & 1.498 & 17 \\
\hline
\end{tabular}

Table 1

Maximal allowable delay $h_{\max }$ for system (33).

\begin{tabular}{|c|c|c|c|}
\hline Theorems & first interval & second interval & $\mathrm{nv}$ \\
\hline \hline$[2]$ & {$[0,0.964]$} & {$[1.372,2.105]$} & - \\
\hline$[24]$ & {$[0,0.964]$} & - & 3 \\
\hline Th.4 $(N=1)$ & {$[0,0.91]$} & - & 8 \\
\hline Th.4 $(N=2)$ & {$[0,0.963]$} & {$[1.382,2.100]$} & 12 \\
\hline Th.4 $(N=3)$ & {$[0,0.964]$} & {$[1.372,2.103]$} & 17 \\
\hline Th.4 $(N=4)$ & {$[0,0.964]$} & {$[1.372,2.105]$} & 23 \\
\hline
\end{tabular}

\section{Table 2}

Intervals of stability w.r.t. $h$ for system (34). The notation "nv" denotes the number of decision variables.

\section{Examples}

Example 1: Let us consider a first scalar example

$$
\dot{x}(t)=-x(t)+\int_{-h}^{0} e^{-\theta} \sin (\theta) x(t+\theta) \mathrm{d} \theta .
$$

Using a numerical method [1,2], system (33) is shown to be asymptotically stable for all delays less than 1.498. Table 1 shows results obtained with Theorem 4 . As expected, better results are obtained as the degree of the polynomial $N$ increases. Moreover, the theoretical upper bound of the delay is recovered with $N=3$.

Example 2: Consider the distributed delay system

$$
\dot{x}(t)=-2 x(t)+\int_{-h}^{0}(\theta+3 \cos \theta) x(t+\theta) \mathrm{d} \theta .
$$

This example is interesting because it is stable for all $h$ in $[0,0.964]$ and in $[1.372,2.105]$ and unstable otherwise [2]. Table 2 shows results obtained with Theorem 4 . Note that even if [24] provides a very good estimation of the first interval of stability, it is not able to detect the second one.

Example 3: Consider the distributed delay system

$$
\dot{x}(t)=-a x(t)-\int_{-h}^{0} \gamma(k, \alpha,-\theta) x(t+\theta) \mathrm{d} \theta,
$$




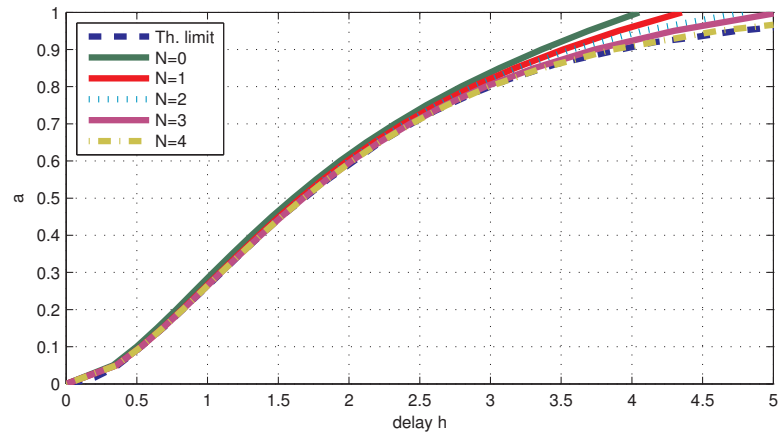

Fig. 1. Stability regions in the plan $(a, h)$ for system (35), obtained using Theorem 4 for several values of $N$.

where $a$ is a positive scalar and $\gamma$ the scalar kernel function of the truncated Gamma Distribution defined by

$\gamma(k, \alpha, \theta)=\frac{\theta^{k-1} e^{-\theta / \alpha}}{(k-1) ! \alpha^{k}}, \forall(k, \alpha, \theta) \in \mathbb{N}_{>0} \times \mathbb{R}_{>0} \times[-h, 0]$,

and such that $\int_{-\infty}^{0} \gamma(k, \alpha, \theta) \mathrm{d} \theta=1$. From the theoretical point of view, Gamma distributions are often considered over the interval $(-\infty, 0]$. Since the kernel $\gamma$ contains an exponential term, it is reasonable to consider the truncated interval $[-h, 0]$ because the main contribution to the distributed term relies on this first interval. Figure 1 represents the stability regions obtained by solving Theorem 4 for several values of $N$ when $\alpha=1$ and $k=1$. The dashed black line represents the theoretical limits resulting from the eigenvalue analysis issued from [2]. Figure 1 shows that from small values of $a$ in $(0,0.6]$, Theorem 4 with $N=0,1$ delivers good estimations of the stability regions. However for larger values of $a$, Figure 1 shows that Theorem 4 with $N=0,1$ is conservatism since the stability regions do not match with the theoretical limits drawn by the dashed blue line. However, increasing $N$ in Theorem 4 allows reducing this conservatism and one can see that for $N=4$, the estimation of the stability region is very close to the theoretical region. This example illustrates the potential of our hierarchical approach to reduce the conservatism by increasing the LMI parameter $N$, of course at the price of increasing the complexity of the conditions, showing the tradeoff between conservatism and complexity.

Example 4: Consider the linear time-delay systems (1) taken from [3]

$$
\dot{x}(t)=\left[\begin{array}{cc}
0.2 & 0 \\
0.2 & 0.1
\end{array}\right] x(t)+\left[\begin{array}{cc}
-1 & 0 \\
-1 & -1
\end{array}\right] \int_{-h}^{0} x_{t}(s) \mathrm{d} s
$$

An eigenvalue analysis provides that the system remains stable for all constant delays in the interval [0.200, 2.04]. In [26], [3] and [19], stability is guaranteed for delays over the interval $[0.2090,1.1942],[0.2001,1.6339]$ and $[0.2001,1.877]$, respectively. Using our new inequality,
Theorem 4 ensures stability for any constant delay $h$ in the intervals [0.2001, 1.58], [0.2001, 1.83], [0.2001, 1.95], $[0.2001,2.02]$, for $N=1,2,3,4$, respectively, which encompasses these previous results. This demonstrates again the potential of Theorem 4 .

Example 5: Consider the system with pointwise delay taken from [4] given by

$$
\ddot{x}(t)+\left[\begin{array}{cc}
0 & 1 \\
-1 & 0
\end{array}\right] \dot{x}(t-h)+\left[\begin{array}{cc}
4 & 0 \\
0 & 16
\end{array}\right] x(t)=0
$$

The analysis provided in [4] based on a frequency method ensures that this systems has exactly three stable delay intervals $[0.4108,0.7509],[2.054,2.252]$ and $[3.697,3.754]$. Figure 2 shows the inner approximations obtained with Theorem 7 for several values of $N$. The first interval is detected at $N=4$ and a good ${ }^{6}$ estimation of the first stable interval is first obtained at $N=7$. First values of the delay $h$ in the second stable interval are detected with $N=9$ and a good estimation of the whole interval is obtained at $N=11$. Delay values in the third and last stable interval, which is more difficult to detect are found with $N=14$ and a good estimation of the interval is provided with $N=16$.

This example shows the efficiency of our method to detect stability intervals even for systems with discrete delay and with dimension that are not small unlike usual examples. In addition, The example and Figure 2 also illustrate Theorem 8. Indeed, Figure 2 shows that the stability regions become larger when $N$ increases. Theorem 7 is able to assess stability of the system for all the values of the delay which belongs to the stable intervals.

\section{Conclusions}

In this paper, we have presented a new LyapunovKrasovskii approach to assess stability of distributed delay systems. Our method is based on a polynomial approximation of the kernel of the distributed delay. The stability analysis is performed thanks to a new set of integral inequalities, which includes Jensen and Wirtinger-based inequalities as particular cases of order 0 and 1. Several stability theorems are provided to cope with distributed delay systems with one or two kernels and also with systems with constant pointwise delay. The efficiency of the method has been successfully tested on several examples.

This work opens a new direction of research regarding the various possibilities to exploit the Bessel inequality with other sequences of orthogonal functions such as trigonometric functions, Chevishev or Laguerre polynomials, which would potentially allow to design a wider class of Lyapunov-Krasovskii functionals.

\footnotetext{
$\overline{6}$ with a precision of $10^{-4}$
} 


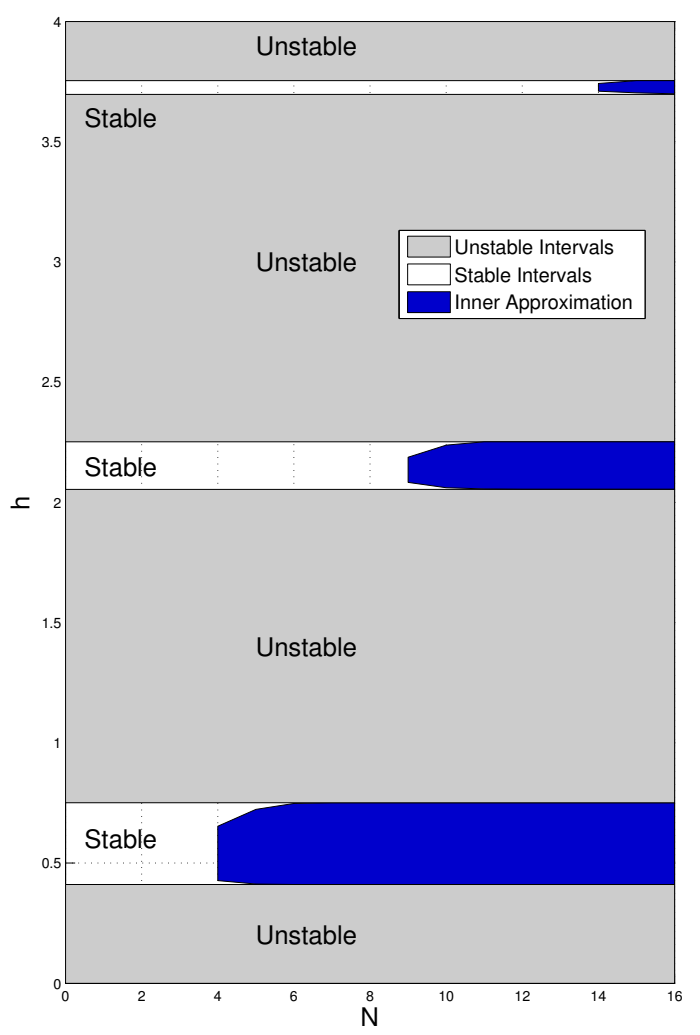

Fig. 2. Stability region in the plan $(N, h)$, obtained using Theorem 7 .

\section{References}

[1] D. Breda, S. Maset, and R. Vermiglio. Pseudospectral differencing methods for characteristic roots of delay differential equations. SIAM Journal on Scientific Computing, 27(2):482-495, 2005.

[2] D. Breda, S. Maset, and R. Vermiglio. Trace-dde: a tool for robust analysis and characteristic equations for delay differential equations. In Topics in Time Delay Systems, volume 388 of Lecture Notes in Control and Information Sciences, pages 145-155. Springer Berlin Heidelberg, 2009.

[3] W.-H. Chen and W.X. Zheng. Delay-dependent robust stabilization for uncertain neutral systems with distributed delays. Automatica, 43(1):95 - 104, 2007.

[4] P. Freitas. Delay-induced instabilities in gyroscopic systems. SIAM Journal on Control and Optimization, 39(1):196-207, 2000.

[5] E. Fridman and U Shaked. A descriptor system approach to $\mathcal{H}_{\infty}$ control of linear time-delay systems. IEEE Trans. on Automatic Control, 47:253-270, February 2002.

[6] W. Gautschi. Orthogonal Polynomials, Computation and Approximation. Oxford Science, 2004.

[7] F Gouaisbaut and Y Ariba. Delay range stability of a class of distributed time delay systems. Systems $\&$ Control Letters, 60(3):211-217, 2011.

[8] K. Gu, Q.L. Han, A.C.J. Luo, and S.I. Niculescu. Discretized lyapunov functional for systems with distributed delay and piecewise constant coefficients. International Journal of Control, 74(7):737 - 744, 2001.
[9] K. Gu, V. L. Kharitonov, and J. Chen. Stability of Time-Delay Systems. Birkhäuser Boston, 2003. Control engineering.

[10] Q.-L. Han. A descriptor system approach to robust stability of uncertain neutral systems with discrete and distributed delays. Automatica, 40(10):1791 - 1796, 2004.

[11] V. B. Kolmanovskii and L. E. Shaikhet. Control of systems with aftereffect. American Mathematical Society, 1996.

[12] X.-G. Li and X.-J. Zhu. Stability analysis of neutral systems with distributed delays. Automatica, 44(8):2197 - 2201, 2008.

[13] C.-I. Morarescu, S.-I. Niculescu, and Gu K. Stability crossing curves of shifted gamma-distributed delay systems. SIAM Journal on Applied Dynamical Systems, 6(2):475-793, 2007.

[14] U. Münz, J. Rieber, and F. Allgöwer. Topics in TimeDelay Systems: Analysis, Algorithms, and Control, volume 388 of $L N C I S$, chapter Robust Stabilization and $\mathcal{H}_{\infty}$ Control of Uncertain Distributed Delay Systems, pages 221-231. Springer, Berlin, 2009.

[15] S.I. Niculescu. Delay Effects on Stability. A Robust Control Approach, volume 269 of Lecture Notes in Control and Information Sciences. Springer-Verlag, Heildelberg, 2001.

[16] H. Ozbay, C. Bonnet, and J. Clairambault. Stability analysis of systems with distributed delays and application to hematopoietic cell maturation dynamics. In Decision and Control, 2008. CDC 2008. 47th IEEE Conference on, pages 2050-2055, Dec 2008.

[17] M.M. Peet, A. Papachristodoulou, and S. Lall. Positive forms and stability of linear time-delay systems. SIAM Journal on Control and Optimization, 47(6):3227-3258, 2009.

[18] O. Santos, S. Mondie, and V.L. Kharitonov. Robust stability conditions for systems with distributed delays. In Decision and Control, 2006 45th IEEE Conference on, pages 217-222, Dec 2006.

[19] A. Seuret and F. Gouaisbaut. Wirtinger-based integral inequality: Application to time-delay systems. Automatica, 49(9):2860-2866, 2013.

[20] A. Seuret and F. Gouaisbaut. Hierarchy of LMI conditions for the stability of time delay systems. Systems \& Control Letters, 81:1-7, 2015.

[21] A. Seuret, F. Gouaisbaut, et al. Complete quadratic Lyapunov functionals using Bessel-Legendre inequality. Proceeding of the 13th European Control Conference, 2014.

[22] R Sipahi, F Atay, and S Niculescu. Stability of Traffic Flow Behavior with Distributed Delays Modeling the Memory Effects of the Drivers. SIAM Journal on Applied Mathematics, 68(3):738-759, 2008.

[23] R. Sipahi, S. Niculescu, C.T. Abdallah, W. Michiels, and Keqin Gu. Stability and stabilization of systems with time delay. Control Systems, IEEE, 31(1):38 -65, feb. 2011.

[24] O. Solomon and E. Fridman. New stability conditions for systems with distributed delays. Automatica, 49(11):3467 $3475,2013$.

[25] L. Xie, E. Fridman, and U. Shaked. A robust $\mathcal{H}_{\infty}$ control of distributed delay systems with application to combustion control. IEEE Trans. on Automatic Control, 46(12):19301935, 2001.

[26] F. Zheng and P.M. Frank. Robust control of uncertain distributed delay systems with application to the stabilization of combustion in rocket motor chambers. Automatica, (3):487-497, 2002. 